\title{
Distinguishing childhood absence epilepsy patients from controls by the analysis of their background brain electrical activity
}

\author{
Osvaldo A. Rosso ${ }^{\mathrm{a}, \mathrm{b}}$, Alexandre Mendes ${ }^{\mathrm{a}}$, John A. Rostas ${ }^{\mathrm{c}}$, \\ Mick Hunter ${ }^{\mathrm{d}}$ and Pablo Moscato ${ }^{\mathrm{a}}$ \\ ${ }^{a}$ Centre for Bioinformatics, Biomarker Discovery and Information-Based \\ Medicine and Hunter Medical Research Institute. \\ School of Electrical Engineering and Computer Science, \\ The University of Newcastle, University Drive, Callaghan, NSW, 2308, Australia. \\ ${ }^{\mathrm{b}}$ Instituto de Cálculo, Facultad de Ciencias Exactas y Naturales, \\ Pabellón II, Ciudad Universitaria, \\ 1428 Ciudad Autonoma de Buenos Aires, Argentina. \\ ${ }^{\mathrm{c}}$ Biomedical Sciences and Hunter Medical Research Institute, \\ The University of Newcastle, University Drive, Callaghan, NSW, 2308, Australia. \\ ${ }^{\mathrm{d}}$ School of Psychology and Hunter Medical Research Institute, \\ The University of Newcastle, University Drive, Callaghan, NSW, 2308, Australia.
}

\begin{abstract}
Background electroencephalography (EEG), recorded with scalp electrodes, in children with childhood absence epilepsy (CAE) and control individuals has been analyzed. We considered 5 CAE patients, all right-handed females and aged 6-8 years. The 15 control individuals had the same characteristics of the CAE ones, but presented a normal EEG. The EEG was obtained using bipolar connections from a standard 10-20 electrode placement $(F p 1, F p 2, F 7, F 3, F z, F 4, F 8, T 3, C 3, C z$, $C 4, T 4, T 5, P 3, P z, P 4, T 6, O 1$ and $O 2)$. Recordings were undertaken in the resting state with eyes closed. EEG hallmarks of absence seizure activity are widely accepted, but there is a recognition that the bulk of interictal EEG in CAE appears normal to visual inspection. The functional activity between electrodes was evaluated using a wavelet decomposition in conjunction with the Wootters distance. Then, pairs of electrodes with differentiated behavior between CAE and controls were identified using a test statistic-based feature selection technique. This approach identified clear differences between CAE and healthy control background EEG in the frontocentral electrodes, as measured by Principal Component Analysis. The findings of this pilot study can have strong implications in future clinical practice.
\end{abstract}

Key words: EEG, background, absence epilepsy, classification PACS: 87.80Tq, 05.45.Tp, 87.70.Cf 


\section{Introduction}

The electroencephalogram (EEG) is a representative signal containing information about the condition of the brain. In fact, the oscillations of the EEG may contain useful information about the state of the brain and how it works (Başar 1980, 1998, 1999). We can also accept as working hypothesis that the EEG recorded under resting conditions is representative of the global state of the brain. This point of view is supported by the implicit assumption that different brain regions are governed by the same dynamic process if they are not activated by a particular task. As a consequence, we can expect that individual patterns might become prominent (Elbert et al., 1992). Then, another plausible working hypothesis is that EEG time series (background EEG) corresponding to healthy controls differs from patients with different pathologies (e.g. epilepsy) or neurodegenerative diseases (e.g. schizophrenia, Alzheimer). Moreover, the same hypothesis can be applied to discover and specify electrocortical substrates for cognitive, emotional, and behavioural conditions.

Analysis of EEG signals always involves the queries of quantification, i.e., the ability to state objective data in numerical and/or graphical form. Without such measures, EEG appraisal remains subjective and hardly lead to logical systematization (Niedermeyer and Lopes da Silva, 1987). Processing of information by the brain is reflected in dynamical changes of electrical activity in time, frequency and space. Therefore, study of this process requires methods which can describe the variation of the signal in time and frequency. An attractive property for these quantifiers is that they are related with physical properties; therefore their interpretation and the implications of their results is straightforward. As a result, to understand the associated dynamics of the EEG time series, one can study the temporal evolution of these associated quantifiers and reach conclusions about their behavior under different pathologies and diseases.

Very interesting results characterizing the time evolution of EEG associated dynamics have been reported by some research groups using quantifiers based on measurements of nonlinear dynamics applied to EEG epileptic time series provided by depth and cortical electrodes. They analyze the EEG during seizure onset and the background EEG (pre- and post-ictal), following the temporal evolution of the signal complexity (associated with the measurement of the correlation dimension $D_{2}$ ) (Casdagli et al., 1997; Lehnertz and Elger, 1998) and the chaoticness degree (through the largest Lyapunov exponent, $\Lambda_{\max }$ ) (Iasemeddis et al. 1990; Iasemedis and Sakellares, 1991; Kowalik and Elbert, 1994). The main findings from these reports are a reduction of 
these two quantifiers during the ictal stage, suggesting that a transition takes place at the seizure onset in the dynamic behavior of the neural network from a complex behavior to a simpler one (Pijn et al. 1991). Furthermore, some researchers report a significant decrease of these quantifiers a few minutes before the seizure-onset raising the hypothesis that epileptic seizures could be predicted (Iasemeddis et al. 1990; Iasemedis and Sakellares, 1991; Lehnertz and Elger, 1998). The basic requirement for nonlinear dynamic metric tools (Chaos Theory) to be applied to experimental data is the stationarity of the time series, which suggests the time series is representative of a unique and stable attractor. Also for the evaluation of $D_{2}$ and $\Lambda_{\max }$, defined as asymptotic properties of the attractor, long time recordings are required. By applying a static measurement of $D_{2}$ and $\Lambda_{\max }$ to selected portions of brain electrical activity (EEG time series), satisfying all the mathematical hypothesis requirement, different states can be characterized (Babloyantz et al., 1985; Babloyeantz and Destexhe, 1986; Blanco et al., 1997a,b; 2003; Rosso et al. 2004). These metric invariants require the computation of some previous parameters which rapidly degrade with additive noise, often giving vague results (for a review see Elbert et al., 1994 and Başar, 1998).

Another group of qEEG methods are based on time-frequency analysis, i.e. evaluating the Gabor or Wavelet Transform of the corresponding EEG signal (Blanco et al., 1995; 1996; 1998; Rosso et al. 2001; 2006). These techniques allow to study the EEG time series in the time-frequency domain. These methods, specially orthogonal discrete wavelet transform (ODWT), do not make assumptions about record stationarity (they do not need to reconstruct any attractor of the dynamical system) and they work only with their measurable response, that is the time series. Taking the wavelet transform of the EEG signal as a basic element, we can define two quantifiers: the relative wavelet energy (RWE) and the wavelet entropy (WS). The RWE provides information about the relative energy associated with the different frequency bands present in the EEG and their corresponding degree of importance. WS bring us information about the degree of order/disorder associated with a multi-frequency signal response. Considering that, under some hypotheses concerning in the nature of nonlinear systems, Lyapunov exponents and Shannon entropy concepts can be related (Pesin, 1977; Eckmann and Ruelle, 1985) the time evolution of these quantifiers, RWE and WS, could give information about the dynamics associated with the EEG records (Rosso and Mairal, 2002).

The application of nonlinear dynamics (e.g. see the reviews of Stam (2005) and Pritchard et al. (1995), and reference therein); Information Theory (Tong et al., 1996; Xu et al., 1997; Jeong et al., 2001; Na et al., 2002; Kannathal et al., 2005; Gómez et al., 2006, 2007; Abásolo et al., 2006; Fernández et al., 2007; Figliola et al., 2007; Montani et al., 2007a, 2007b); and time-frequency analysis (Rosso and Mairal, 2002; Rosso et al., 2001, 2006; Rosso, 2007), among other novel techniques have opened up a range of new perspectives for the study of 
normal and disturbed brain functions using background EEG.

It is currently accepted that a human observer cannot discriminate EEG traces of healthy controls from schizophrenics (Elbert et al., 1992) or interictal epileptic activity (Jan, 2002) - just to mention some cases. Quantitative EEG analysis using computational methods can thus assist in the background EEG characterization. To our knowledge, there has been only one quantitative EEG analysis to differentiate background signals between controls and epileptic individuals. In 2005, Rosso et al. published a single-channel analysis of EEG signals which was based on wavelet entropy. This work represents a further step, where we used wavelet decomposition combined with multi-channel analysis.

Epilepsy is a neurological disorder that affects millions of patients worldwide and arises from the concurrent action of multiple pathophysiological processes. Childhood epilepsies are different from many adult epilepsies in that onset and offset are often determined by age, i.e. there is an important influence of, or link with, maturity. Archetypical age-related epilepsies would include the two best described childhood absence epilepsy syndromes: childhood absence epilepsy (CAE) and juvenile absence epilepsy (JAE). Whilst both these epilepsies resemble each other in important aspects, they differ in age at presentation, indicating an important relationship with development.

Undoubtedly, the EEG constitutes a very important clinical tool in investigating children with various neurological disorders, particularly epilepsy. Indeed, even when the diagnosis of seizures and epileptic syndromes are primarily clinical, the EEG records provide supportive evidence and helps in the seizure classification (Sundaram et al., 1999). Absence epilepsy is characterized by generalized non-convulsive epileptic seizures expressed predominantly as disturbances of consciousness with no, or relatively little motor activity (Aicardi, 1994; Holmes, 1997). Day dreaming in class and at home in children is a major source of referral to the hospital for consideration of absence seizures. Typical absence seizures take place in otherwise apparently normal children and adults, and have an EEG hallmark of brief ictal and inter-ictal 3-3.5 $\mathrm{Hz}$ spike-and-wave discharges with a maximum amplitude over the fronto-rolandic regions. The EEG background is otherwise normal to standard visual analysis in the vast majority of cases (Blume et al., 1999; Wyllie, 1991; Aicardi, 1994; Holmes, 1997; Jan, 2002) and as consequence is disregarded by the clinicians for diagnostic purposes (Jan, 2002). Given the strong link with development in absence epilepsy in terms of onset and sometimes remission, it may be that the quantitative background EEG differs from those of unaffected children of the same age.

In previous work by recourse of the normalized total wavelet entropy (NTWS) (Rosso and Mairal, 2002; Rosso et al., 2001, 2006; Rosso, 2007), we examined 
the background EEG of those patients demonstrating absence seizure activity, and compare it with children without absence epilepsy (Rosso et al., 2005). The NTWS can be used to define the degree of disorder (chaos) in the brain functional sources. Higher entropy values $(0 \leq N T W S \leq 1)$ means higher dynamical complexity, higher irregular behaviour and, of course, lower predictability. In this single channel analysis, entropy values (NTWS) were calculated for patients versus controls. For all channels, combined background EEG of patients with absence epilepsy showed (statistically significant) lower entropy values than controls. The size of the difference in entropy values was not uniform, with certain EEG electrodes consistently showing greater differences than others. The NTWS calculation demonstrates that the inter-ictal activity in absence epilepsy patients does differ from controls. As expected, the entropy values for the seizure epochs were lower than during the background epochs. One can associate a more robust degree of order to the functional networks during the ictal than during the pre- and post-ictal stages, compatible with a dynamic process of synchronization in the brain activity (Rosso and Mairal, 2002; Rosso et al., 2001, 2006; Rosso, 2007; Stam, 2005).

In the present work, we combine the scalp EEG signal (channel) wavelet decomposition with a feature selection method. The Relative Wavelet Energy (RWE) is used in order to evaluate the inter channel similarity activity matrices, that represent the functional connectivities (Stam, 2005). Their quantification is made by a feature selection based on a Kruskal-Wallis statistical test for class differentiation. The goal was to find a clear differentiation of the functional networks between EEG background corresponding to a sample set of child patients presenting CAE and healthy control individuals, and also to identify which set of electrodes provide the maximum differentiation. The analysis procedure provides a clear discrimination between groups, finding patterns of functional networks (cerebral activity) which can be used to diagnose CAE and thus have an impact in future clinical practice.

\section{Materials and Methods}

\subsection{Materials}

Cases for this pilot study were selected by searching in the 'history' field of the John Hunter Hospital EEG Database, Newcastle, Australia (Hunter et al., 2005) for children referred for the investigation of absence epilepsy. Inclusion criteria were a history of normal cognition, with absence seizures only, and free of anticonvulsant, sedation and other medications. EEG inclusion criteria were normal EEG background to standard clinical perusal. 
The eligible cases were further restricted to right-handed females, aged between 6 and 8 years. The patient group of 5 patients had a mean age of 83 months, and the control group (same inclusion criteria as patients, but with a normal EEG) contained 15 patients with a mean age of 81 months. The EEG was obtained using bipolar connections from a standard 10-20 electrode placement $(F p 1, F p 2, F 7, F 3, F z, F 4, F 8, T 3, C 3, C z, C 4, T 4, T 5$, $P 3, P z, P 4, T 6, O 1$ and $O 2)$. Recordings were undertaken in the resting state with eyes closed. In this way it may be assumed that different brain regions are governed by the same dynamic process. All EEGs were selected and analyzed by an experienced pediatric neurologist. In all 20 cases chosen for this study, two or more $1 \mathrm{~min}$ epochs of artifact-free background EEG were selected, depending on availability. For the patient group, one or two seizure epochs were marked (also depending on availability of such events) in addition to the background epochs. These epochs (ictal and non-ictals) were then exported as ASCII files for subsequent analysis. The number of time windows (see next section) used in the computational tests in this work - Feature Selection and Principal Component Analysis - were 41 for controls background, 15 for CAE patients background and 8 representing seizures.

\subsection{Wavelet transform, wavelet quantifiers and Wootters distance}

Orthogonal decimated discrete wavelet transform (ODWT) was applied to EEG signals $\mathcal{S}(t) \equiv\left\{s_{n}, n=1, \cdots, M\right\}$, in which orthogonal cubic spline functions were used as mother wavelets $\psi_{j, k}(t)$ with $j, k \in \mathbb{R}$ the scale and translation indexes, and the time-frequency information was organized in a hierarchical scheme (Rosso and Mairal, 2002; Rosso et al., 2001, 2006; Rosso, 2007). Seven frequency bands for an appropriate wavelet analysis within the multiresolution scheme were defined $(j=-7, \cdots,-1$; wavelet resolution levels) according to the signal sample frequency $\left(\omega_{\mathrm{s}}=167 \mathrm{~Hz}\right)$.

Each EEG signal $\mathcal{S}$ and the corresponding wavelet coefficients series $C_{j}(k)=$ $\left\langle\mathcal{S}, \psi_{j, k}\right\rangle$ are divided into non-overlapping epochs of length $L=128$ samples and for each time window $i\left(i=1, \cdots, N_{T}=M / L\right)$ the energy probability distribution, called the Relative Wavelet Energy (RWE) and Normalized Total Wavelet Entropy (NTWS) are computed (Rosso and Mairal, 2002; Rosso et al., 2001, 2006; Rosso, 2007). Since the family $\psi_{j, k}(t)$ is an orthonormal basis for $L^{2}(\mathbb{R})$, the concept of energy is linked to the usual notions derived from Fourier Analysis. The wavelet energy at resolution level $j$ for time window $i$ is evaluated as

$$
E_{j}^{(i)}=\sum_{k=(i-1) \cdot L+1}^{i \cdot L}\left|C_{j}(k)\right|^{2} \quad \text { with } \quad i=1, \cdots, N_{T}
$$


and the corresponding total energy in this time window will be

$$
E_{\text {Tot }}^{(i)}=\sum_{j=J_{M}}^{J_{0}} E_{j}^{(i)} \quad \text { with } \quad i=1, \cdots, N_{T}
$$

where $J_{M}$ and $J_{0}$ are the minimum and maximum wavelet resolution levels included. Then the Relative Wavelet Energy, for the wavelet resolution level $j$, at time window $i$, is defined by

$$
\rho_{j}^{(i)}=E_{j}^{(i)} / E_{\text {Tot }}^{(i)} \quad \text { with } \quad i=1, \cdots, N_{T},
$$

and the associated Normalized Total Wavelet Entropy is

$$
H^{(i)}=-H_{0} \sum_{j=J_{M}}^{J_{0}} \rho_{j}^{(i)} \ln \rho_{j}^{(i)} \quad \text { with } \quad i=1, \cdots, N_{T}
$$

Where $H_{0}=1 / \ln \left|J_{0}-J_{M}+1\right|$ is the normalization constant. In the present work the wavelet quantifiers are computed excluding wavelet resolution levels out of the frequency range $0.65-20.87 \mathrm{~Hz}$, that is $J_{M}=-7$ and $J_{0}=-3$ (for more details, see Rosso et al., 2001, 2006).

The obtained energy probability distribution (RWE) for different EEG signals (channels) can be compared using statistical distances. In this study we evaluate the distance matrix between channels using the Wootters statistical distance (Wootters, 1981). Given the probability wavelet energy distribution (see eq. (3)) $P^{(i)}=\left\{\rho_{j}^{(i)}, j=-N_{J}, \cdots,-j_{0}\right\}$, we defined the normalized Wootters distance between two signals $\alpha$ and $\beta$, at time window $i$, by

$$
D^{(W)}\left[P_{\alpha}^{(i)}, P_{\beta}^{(i)}\right]=\frac{2}{\pi} \cos ^{(-1)}\left[\sum_{j=-N_{J}}^{-j_{0}}\left(\rho_{j}^{(\alpha ; i)} \cdot \rho_{j}^{(\beta ; i)}\right)^{(1 / 2)}\right]
$$

Following the definitions introduced by C.J. Stam (2005):

- Functional Source is the part or parts of the brain that contribute to the activity recorded at single sensor. It is a shorthand for denoting the part of the brain being measured at single recording site and constitute the lowest level of spatial resolution.

- Functional Connectivity is defined as any correlation between the activity of two functional sources.

- Functional Network is represented by the full matrix of all pair-wise correlations between functional sources. 
Combined with the Wavelet Transform properties, the previously defined wavelet quantifiers (see Eqs. (3), (4) and (5)) can be understood as follows. Since the ODWT represents each frequency band with optimal time resolution, the RWE provides information about the relative energy associated with the different frequency band in the EEG and their corresponding degree of importance, as well as, their timing. The RWE is a measure of the energy probability distribution associated with the different functional sources considered. The corresponding wavelet entropy (NTWS) given by the normalized Shannon entropy, provides a measure of the order/disorder state of the functional source and consequently information about the underlying dynamical process. The functional connectivity is evaluated using the Wootters distance (see Eq. (5)) and the functional network is given by the full matrix of all signal pairs (electrode $\equiv$ funtional source). Taken into account that NTWS provides also a measure of the frequency toning at the functional source, then the quantification of the functional network could give us information about the global synchronization degree of the brain, at least at the pair-wise correlation level.

\subsection{Feature selection and Principal Component Analysis}

The resulting data after the wavelet transformation was composed of a series of matrices containing the Wootters distance between pairs of EEG signals for the several control/patient subjects and corresponding time windows. Using this information, we applied a Kruskal-Wallis test to each pair of electrodes, which measured the null hypothesis that the distribution of the Wootters distances in the two groups of individuals are the same. For the feature selection, each pair of electrodes was considered as a feature, which were then ordered following the increasing sequence of $p$-values. To find the subset of features that yields the best separation between the two groups, we used a Principal Component Analysis (PCA) approach.

First, suppose that a PCA was calculated for all individuals/time windows, using a given set of pairs of electrodes. Let $P_{h}=\left\{p_{h}^{1}, p_{h}^{2}, \cdots, p_{h}^{|h|}\right\} ; p_{h}^{i}=\left(x_{h}^{i}, y_{h}^{i}\right)$ be the set of points representing the $|h|$ healthy controls samples. Analogously, let $P_{e}=\left\{p_{e}^{1}, p_{e}^{2}, \cdots, p_{e}^{|e|}\right\} ; p_{e}^{j}=\left(x_{e}^{j}, y_{e}^{j}\right)$ be the $|e|$ points representing the CAE samples. Notice that the axes $x$ and $y$ are now the first and second eigenvectors associated to the PCA, respectively.

The separation between the groups of healthy controls and CAE patients was calculated in two different ways. First, a simple normalized distance, calculated over the first component axis, between the two closest points belonging to 
different groups:

$$
S_{x}=\frac{\min _{i}\left(x_{e}^{i}\right)-\max _{j}\left(x_{h}^{j}\right)}{\max _{i, j}\left(x_{e}^{i}, x_{h}^{j}\right)-\min _{i, j}\left(x_{e}^{i}, x_{h}^{j}\right)}
$$

Equation (6) measures the separation between the two classes of individuals by initially determining the closest pair of points $\left(p_{e}^{i}, p_{h}^{j}\right)$, considering just the first eigenvalue axis ( $x$-axis). Then it subtracts the $x$ coordinates between $p_{e}^{i}$ and $p_{h}^{j}$ and normalizes it by the interval between the minimum and maximum values that occur in the $x$-axis.

The second separation measure takes into account both the first and second components axes ( $x$ and $y$ axis). It was used first in a study of clustering which used the Arithmetic Harmonic Cut (AHC) method (Mahata et al., 2006), and is stated as:

$$
S_{A H C}=\left(\sum_{e \in E_{\text {out }}} w(e)\right)\left(\sum_{e \in E_{\text {in }}} \frac{1}{w(e)}\right)
$$

Consider a complete graph $G(V, E, w)$, where each point in $\left(P_{h} \cup P_{e}\right)$ is mapped into a vertex in $V=\left\{v_{1}, v_{2}, \cdots, v_{|e|+|h|}\right\} ; E=\left\{e_{i j}\right\}$ is the set of all edges connecting all pairs of vertices; and $w=\left\{w_{i j}\right\}$ is the weight of each edge, corresponding to the Euclidean distance between the points mapped into $v_{i}$ and $v_{j}$, in the subspace defined by the two first eigenvectors of the PCA. That said, $E_{\text {out }}$ is the set of edges that connect all pairs of vertices mapped into different groups - i.e. healthy and CAE. Analogously, $E_{i n}$ is the set of edges connecting all pairs of vertices mapped into the same group. Thus, $S_{A H C}$ quantifies how compact and distant from each other are the two groups of points. For a detailed description of this separation measure, along with a discussion about its use, please refer to Mahata et al. (2006).

The rationale of the use of this objective function can be clear if the terms are rearranged and written as:

$$
S_{A H C}=\frac{A_{\text {out }}}{H_{\text {in }}}\left(|E|-\left|E_{\text {out }}\right|\right)\left|E_{\text {out }}\right|
$$

where $A_{\text {out }}$ is the arithmetic mean of the weights that connect vertices of $S$ with $V \backslash S$ (the cut); $H_{i n}$ is the harmonic mean of the weights of the edges not in the cut, and $\left|E_{\text {out }}\right|$ is the cardinality of the cut. Informally, maximizing $S_{A H C}$ is equivalent to try to find a cut that discriminates well the two groups, normalized by the harmonic mean of the intra-cluster dissimilarity, and multiplied by a factor that is maximum when the two groups have a similar number of elements. Normalizing by the harmonic mean allows the denominator being 
more stable to the presence of outlier points when associated to either $V$ or $V \backslash S$. For this reason, this partition was denoted as arithmetic-harmonic cut.

It is reasonable to assume that the $S_{A H C}$ will be less affected by the presence of outliers, as it uses a weighted summation of the distances between points, and not minimum and maximum values as does the $S_{x}$. However, that comes with a cost associated, which in this case is the complexity to implement the $S_{A H C}$; considerably higher than the $S_{x}$.

We should emphasize that the numerical values of separation given by Eqs. (6) and (7) are not comparable to each other. $S_{x}$ will yield separation values that are numerically small, and negative whenever there is overlap of samples from different classes in the $x$-axis of the PCA. $S_{A H C}$ on the other hand will always return positive values, since it is calculated as the product between two sums of positive elements - the Euclidean distances between pairs of points in the PCA. Both separations are thus in different numerical scales. However, $S_{x}$ and $S_{A H C}$ can and should be used independently to verify if certain configurations of pairs of electrodes yield good separations using both measures. That said, high quality subsets of features to separate CAE patients and controls are characterized by high values of separation using both $S_{x}$ and $S_{A H C}$.

\section{Computational Results}

\subsection{Wavelet transformation}

As mentioned before, a Wavelet transformation was applied over the EEG signals and then the Wootters distance between each pair of electrodes signals was calculated for each time window considered. Visual inspection of signals are considerably similar between backgrounds. A visual inspection of the matrices containing the Wootters distances (similarity matrix representing the state of brain functional network) for each control and CAE patient indicates slight differences between the two types of background signals, and a clearer difference between backgrounds and a seizure event.

In Figure 1(a-c), we show the average over the time windows considered which characterizes an epoch - of the functional network, that is the pixelmatrix representation of the distances between EEG channels for three typical control individuals, CAE patients and seizure events. Each graphic corresponds to the average of the time-windows within a specific epoch that was chosen to be representative of the physiological state. Darker pixels indicate smaller distances (high similarity between EEG signals), which identify the active functional networks in the corresponding state. From the Information 
Theory point of view, the information contained in both channels is similar. Analogously, lighter pixels mean larger distances (low similarity of information between channels). The greatest difference observed is between seizure and background EEGs. However, there is no clear discernible difference between healthy controls and CAE backgrounds, which is the same situation faced by physicians when making a visual analysis of the EEGs. The same applies to Figure 1(d), where we show the average over all time windows for the three types of signals. Notably, the difference between control and CAE patients backgrounds is very small, and both differ considerably from seizure events.

As mentioned before, a previous work has suggested that the normalized total wavelet entropy (NTWS) could measure differences in the brain activity between CAE patients and controls (Rosso et al., 2005). Specifically, CAE patients had (statistically significant) lower entropy values than controls in the frontocentral electrodes. Given that, we proceed our analysis to determine the most differentiated pairs of electrodes between backgrounds using a feature selection approach.

\subsection{Feature selection - best pairs of electrodes}

In this section we focus on finding the best set of pairs of electrodes to differentiate controls from CAE patients, and use an iterative procedure to achieve that. Starting with the two pairs of electrodes with the lowest $p$-values for the Kruskal-Wallis test, calculate the PCA over the entire set of individuals/time windows, measure the resulting separation according to Eqs. (6) and (7), and iteratively increment the number of pairs of electrodes considered in the PCA calculation. In Figure 2, we show the separations $S_{x}$ and $S_{A H C}$ of the samples after PCA calculation, when we vary the number of pairs of electrodes in the interval $[2,26]$. For $S_{x}$, positive values of separation indicate that samples of the two groups do not overlap. The best separation contains 13 pairs of electrodes, namely (F4;Fz), (Fp1;F3), (Fp1;F8), (T5;Fz), (C3;Fz), (F8;T5), $(\mathrm{F} 8 ; \mathrm{Fz}),(\mathrm{F} 3 ; \mathrm{C} 3),(\mathrm{T} 4 ; \mathrm{Fz}),(\mathrm{Fp} 1 ; \mathrm{C} 3),(\mathrm{Fp} 2 ; \mathrm{C} 3),(\mathrm{Fp} 2 ; \mathrm{Fz})$ and $(\mathrm{Fp} 1 ; \mathrm{F} 7)$. Their $p$-values range from $p=2.6 \times 10^{-7}$ to $p=7.9 \times 10^{-6}$. For $S_{A H C}$, the best separation comes with 15 pairs of electrodes, which include all the aforementioned, plus $(\mathrm{Fp} 1 ; \mathrm{Fz})$ and $(\mathrm{Fp} 2 ; \mathrm{T} 4)$. Their $p$-values range from $p=2.6 \times 10^{-7}$ to $p=$ $8.6 \times 10^{-6}$.

The two graphs shown in Figure 2 have very similar profiles, and maximum separation values with 13 and 15 pairs of electrodes, for $S_{x}$ and $S_{A H C}$, respectively. These two configurations of pairs of electrodes contain the same 10 individual electrodes, namely Fp1, Fp2, F3, F4, F7, F8, Fz, C3, T5 and T4. These represent majorally frontocentral electrodes and corroborate previous 
findings in terms of the entropy associated to EEG signals in healthy control and CAE patients (Rosso et al., 2005). A diagram with the pairs of electrodes present in the solutions that maximize both $S_{x}$ and $S_{A H C}$ is shown in Figure 3. It depicts the position of the 20 electrodes used in this study, with lines connecting those pairs of electrodes present in the solutions. The solid lines refer to the pairs of electrodes common to both solutions. The dashed lines indicate those two pairs of electrodes that are present only in the 15-electrode solution, which maximizes $S_{A H C}$. Lines indicate a similar behaviour between the electrodes connected. They are thus an indicative of the functional networks that can differentiate the background of CAE patients and control individuals. It is clear that the greatest differentiation between electrodes is located in the frontocentral region of the brain.

In Figure 4, we show four examples of PCAs with different numbers of pairs of electrodes used in the calculation and how that will affect the separation between the classes. First, in Figure 4a, it is shown the PCA calculated over the individuals/time windows using the two pairs of electrodes with best $p$ values - $(\mathrm{F} 4 ; \mathrm{Fz})$ and $(\mathrm{Fp} 1 ; \mathrm{F} 3)$. Those electrodes are not sufficient to provide a clear separation between the two groups, with some overlapping occurring. The best configurations for the two distance measures $S_{x}$ and $S_{A H C}$ are shown in Figures 4b and 4c, which were calculated with 12 and 15 electrodes, respectively. Notice that both configurations of points are very similar and present a clear division. As we add more pairs of electrodes to the PCA calculation, the separation starts to decay, and with 26 pairs - Figure $4 \mathrm{~d}$ - we have a clear overlap of some of the samples again.

The two separation measures provided a similar outcome, with the same set of 10 electrodes present in the 13 and 15 pairs solutions. We can consider this as an indicative of the robustness of the method proposed. Moreover, these results show the applicability of this methodology to the analysis of EEG signals for diagnosis of CAE. The application of the wavelet transformation and the use of the Wootters distance between EEG signals alone produced slight differences between the three types of signals analyzed (see Figure 1). With the inclusion of feature selection, we were able to pinpoint the electrodes most differentiated and use them to achieve a perfect separation between control and CAE patients backgrounds.

\section{Conclusions}

The EEG signature of typical absence seizure is the sudden onset of 3-3.5 Hz generalized symmetrical spike-and-wave or multiple spike-and-slow wave complexes. The voltage of the discharges is often maximal in the frontocentral regions (Holmes, 1997). The inter-ictal EEG background is often normal in 
typical absence seizures. Our analysis allowed for a more detailed examination of the EEG signal than is possible by visual inspection. Although the background epochs marked were indeed "normal" to standard visual inspection, the proposed methodology based on wavelet informational tools plus the feature selection using $p$-values associated to a Kruskal-Wallis statistical test, demonstrates that the inter-ictal activity in absence epilepsy patients does differ from controls. Moreover, the described methodology constitute a quantitative marker of background EEG maturity between normal children and children with absence epilepsy syndrome and could provide information about maturity in the onset of CAE. Also, the identification of a digital signature for absence or other less distinct epilepsies may enable enhanced detection of those patients who may experience seizures, in the case where a seizure event is not recorded on the first EEG screening.

\section{Acknowledgements}

The authors wish to thank Dr. W. Hyslop and Dr. R.L.L. Smith for their help in the selection and clinical evaluation of the EEG recordings used in this work. This work was partially supported by the Consejo Nacional de Investigaciones Científicas y Técnicas (CONICET), Argentina (PIP 5687/05). O. A. Rosso gratefully acknowledges the support from Australian Research Council (ARC) Centre of Excellence in Bioinformatics, Australia.

\section{References}

Abásolo D, Hornero H, Gómez C, García M, Fernández A, López M. Analysis of EEG background activity in Alzheimer's disease patients with Lempel-Ziv complexity and central tendency measure. Medical Engineering \& Physics 2006; 28: $315-22$.

Aicardi J. International Review of Child Neurology: Epilepsy in Children. New York: Raven Press Books; 1994.

Babloyantz A, Destexhe A. Low dimensional chaos in an instance of epilepsy, Proc. Natl. Acad. Sci. USA 1986; 83: 3513-17.

Babloyantz A, Salazar JM, Nicolis C. Evidence of chaotic dynamics of brain activity during the sleep cycle, Phys. Lett. 1985; 111: 152-6.

Başar E. EEG-Brain Dynamics. Relation Between EEG and Brain Evoked Potentials. Amsterdam: Elsevier; 1980.

Başar E. Brain Function and Oscillations (I): Brain Oscillations, Principles and Approaches. Berlin: Springer; 1998.

Başar E. Brain Function and Oscillations (II): Integrative Brain Function. Neurophysiology and Cognitive Processes. Berlin: Springer; 1999.

Blume WT, Kaibara M. Role of electroencephalogram in some pediatric neurolog- 
ical problems. In: Atlas of Pediatric Eletroencephalgraphy, Blume WT, Keibira M (Editors), Philadelphia: Lippincott Raven; 1999, pp. 361-71.

Blanco S, Creso J, Figliola A, Quian Quiroga R, Rosso OA. Nonlinear dynamic analysis of scalp EEG epileptic signals. In: O. Descalzi, J. Martinez, E. Tirapegui (Eds.), Instabilities and Nonequilibrium Structures VII \& VIII, Kluwer Academic Publishers, Dordrecht, 2003, pp.149-57.

Blanco S, DAttellis C, Isaacson S, Rosso OA, Sirne R, Time-frequency analysis of electroencephalogram series (II): Gabor and wavelet transform, Phys. Rev. E 1996; 54: 6661-72.

Blanco S, Figliola A, Kochen S, Rosso OA. Using nonlinear dynamics metrics tools for characterizing brain structures, IEEE Eng. Med. Biol. Mag. 1997; 16: 83-92.

Blanco S, Figliola A, Quian Quiroga R, Rosso OA, Serrano E. Time-frequency analysis of electroencephalogram series (III): wavelet packets and information cost function, Phys. Rev. E 1998; 57; 932-40.

Blanco S, Kochen S, Quian Quiroga R, Riquelme L, Rosso OA, Salgado P. Characterization of epileptic EEG time series (I): Gabor transform and nonlinear dynamics methods. In: C.E. DAttellis, E.M. Fernandez-Berdaguer (Eds.), Wavelet Theoryand Harmonic Analysis in Applied Sciences, BirkhWauser Publishers, Boston, 1997, pp. 179-226.

Blanco S, Quian Quiroga R, Rosso OA, Kochen S. Time-frequency analysis of electroencephalogram series, Phys. Rev. E 1995; 51: 2624-31.

Casdagli MC, Iasemedis LD, Savit RS, Gilmore RL, Roper SN, Sackellares JC. Non-linearity in invasive EEG recordings from patients with temporal lobe epilepsy, Electroenceph. Clin. Neurophysiol. 1997; 102: 98-105.

Clarke AR, Barry RJ, McCarthy R, Selikowitz M. Age and sex effects in the EEG: development of the normal child. Clinical Neurophysiology 2001; 112: 806-814.

Eckmann J, Ruelle D. Ergodic theory of chaos and strange attractors, Rev. Mod. Phys. 1985; 57: 617-656.

Elbert Th, Lutzenberger W, Rockstroh B, Berg P, Cohen R. Physical aspects of the EEG in schizophrenia. Biol. Psychiatry 1992; 32: 595-606.

Elbert T, Ray WJ, Kowalik ZJ, Skinner JE, Graf KE, Birbaumer N, Chaos and physiology: deterministic chaos in excitable cell assemblies, Physiol. Rev. 1994; 74: 1-47.

Fernández JG, Larrondo HA, Figliola A, Serrano E, Rostas JAP, Hunter M, Rosso OA. Brain maturation changes characterized by Algorithmic Complexity (Lempel and Ziv Complexity). In: XV Conference on Nonequilibrium Statistical Mechanics and Nonlinear Physics. Descalzi O, Rosso OA, Larrondo HA (Editors). American Institute of Physics, AIP Conference Proceedings 2007; 913: 196-202.

Figliola A, Serrano E, Rostas JAP, Hunter M, Rosso OA. Study of EEG brain maturation signals with multifractal detrended fluctuation analysis. In XV Conference on Nonequilibrium Statistical Mechanics and Nonlinear Physics. Descalzi O, Rosso OA, Larrondo HA (Editors). American Institute of Physics, AIP Conference Proceedings 2007; 913: 190-5.

Gómez C, Hornero H, Abásolo D, Fernández A, Escudero J. Analysis of magnetoencephalogram background activity in Alzheimer's disease with auto-mutual information. Computer Methods and Programs in Biomedicine 2007; 87: 239-47. 
Gómez C, Hornero H, Abásolo D, Fernández A, López M. Complexity analysis of the magnetoencephalogram background activity in Alzheimer's disease patients. Medical Engineering \& Physics 2006; 28: 851-59.

Holmes JL. Classification of Seizures and the Epilepsies. In: The Comprehensive Evaluation and Treatment of Epilepsy. Schachter SC, Schomer DL (Editors). San Diego: Academic Press; 1997.

Hunter M, RLL, W. Hyslop, Rosso OA, Gerlach R, Rostas JAP, Williams DB, Henskens F. The Australian EEG database. Clinical EEG and Neuroscience 2005; 36: 76-81.

Iasemedis LD, Sackellares JC. The evolution with time of spatial distribution of the largest Lyapunov exponent on the human epileptic cortex. In: D. Duke, W. Pritchards (Eds.), Measuring Chaos in Human Brain, World Scientific, Singapore, 1991, pp. 49-82.

Iasemedis LD, Sackellares JC, Zaveri HP, Williams WJ. Phase space topography and Lyapunov exponent of electrocorticograms in partial seizures, Brain Topography 1990; 2: 187-201.

Jan MMS. Assessment of the utility of paediatric electroencephalography. Seizure 2002; 11: 99-103.

Jeong J, Gore JC, Peterson BS. Mutual information analysis of EEG in patients with Alzheimer disease. Clinical Neurophysiology 2001; 112: 827-35.

Kannathal N, Choo ML, Rajendra Acharya U, Sadasivan PK. Entropies for detection of epilepsy on EEG. Computer Methods and Programs in Biomedicine 2005; 80: 187-94.

Kowalik ZJ, T. Elbert T. Changes of chaoticness in spontaneous EEG/EMG. Integrative Physiol. Behav. Sci. 1994; 29: 270-82.

Mahata P, Costa W, Cotta C, Moscato P. Hierarchical Clustering, Languages and Cancer. Proceedings of EvoBIO2006 - $4^{\text {th }}$ European Workshop on Evolutionary Computation and Machine Learning in Bioinformatics, Lecture Notes in Computer Science 2006; 3907: 67-78.

Montani F, Kohn A, Smith MA, Schultz SR. The Role of Correlations in Direction and Contrast Coding in the Primary Visual Cortex. The Journal of Neuroscience 2007; 27:2338-48.

Montani F, Rosso OA, Schultz SR. Discrimination Measure of Correlations in a Population of Neurons by Using the Jensen-Shannon Divergence. In XV Conference on Nonequilibrium Statistical Mechanics and Nonlinear Physics. Descalzi O, Rosso OA, Larrondo HA (Editors). American Institute of Physics, AIP Conference Proceedings 2007; 913: 184-89.

Na SH, Jin S, Kim SY, Ham B. EEG in schizophrenic patients: mutual information analysis. Clinical Neurophysiology 2002; 113: 1954-60.

Niedermeyer E, Lopes da Silva FH (Editors). Electroencephalography, Basic Principles, Clinical Applications, and Related Field. Baltimore: Urban \& Schwarzenberg, 1987.

Lehnertz K, Elger CE. Can epileptic seizures be predicted? Evidence from nonlinear time series analysis of brain electrical activity, Phys. Rev. Lett. 1998; 80: 5019-22.

Pesin Y. Lyapunov characteristic exponents and smooth ergodic theory. Russ. Math. Surv. 1977; 32: 55-114. 
Pijn JP, Van Neerven J, Noestt A, Lopes da Silva FH. Chaos or noise in EEG signals: dependence on state and brain site. Electroenceph. Clin. Neurophysiol. 1991; 79: 371-81.

Pritchard WS, Duke DW. Measuring Chaos in the Brain: A Tutorial Review of Nonlinear Dynamical EEG Analysis. International Journal of Neuroscience 1992; 67: 31-80.

Rosso OA. Entropy changes in brain function. International Journal of Pyschophysiology 2007; 64: 75-80.

Rosso OA, Blanco S, Yordanova J, Kolev V, Figliola A, Schürmann M, Basar E. Wavelet entropy: a new tool for the analysis of short duration brain electrical signals. Journal of Neuroscience Methods 2001; 105: 65-75.

Rosso OA, Figliola A, Creso J, Serrano E. Analysis of wavelet-filtered tonic-clonic electroencephalogram recordings. Med. Biol. Eng. Comput. 2004; 42: 516-23.

Rosso OA, Hyslop W, Gerlach R, Smith RLL, Rostas JAP, Hunter M. Quantitative EEG analysis of the maturational changes associated with childhood absence epilepsy. Physica A 2005; 356: 184-9.

Rosso OA, Mairal ML. Characterization of time dynamical evolution of electroencephalographic epileptic records. Physica A 2002; 312: 469-504.

Rosso OA, Martín MT, Figliola A, Keller K, Plastino A. EEG analysis using wavelet-based information tools. Journal of Neuroscience Methods 2006; 153: $163-82$.

Stam CJ, Nonlinear dynamical analysis of EEG and MEG: Review of an emerging field. Clinical Neurophysiology 2005; 116: 2266-301.

Sundaram M, Sadler RM, Young GB, Pillary N. EEG in epilepsy: current perspectives. Canadian Journal of Neurological Sciences 1999; 26: 255-62.

Tong Q, Kong J, Xu J. A note on analyzing schizophrenic EEG with complexity measure. Chaos, Solitons and Fractals 1996; 7: 371-75.

Wyllie E, Friedman D, Luders H. Outcome of psychogenetic seizures in children and adolescents compared to adults. Neurology 1991; 41: 742-44.

Wootters WK. Statistical distance and Hilbert space. Physical Review D 1981; 23: 357-62.

Xu H, Liu Z, Liu R, Yang Q. Information transmission in human cerebral cortex. Physica D 1997; 106: 363-74. 
(a) Typical background signals - Controls
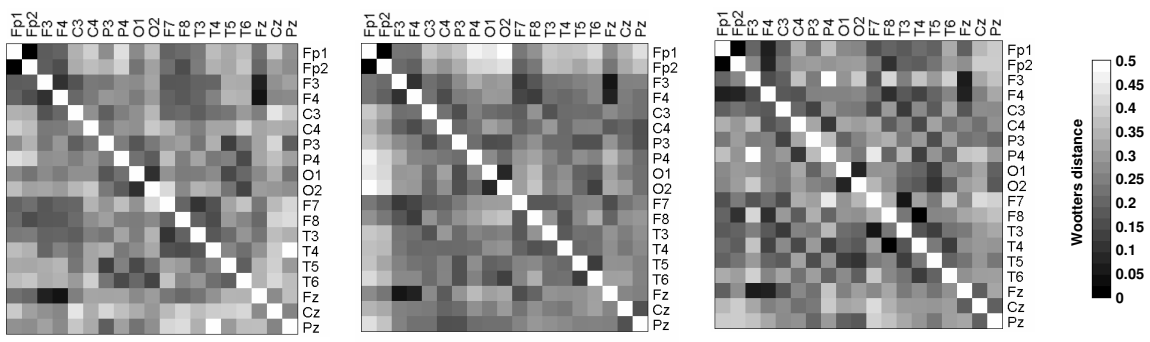

(b) Typical background signals - CAE patients
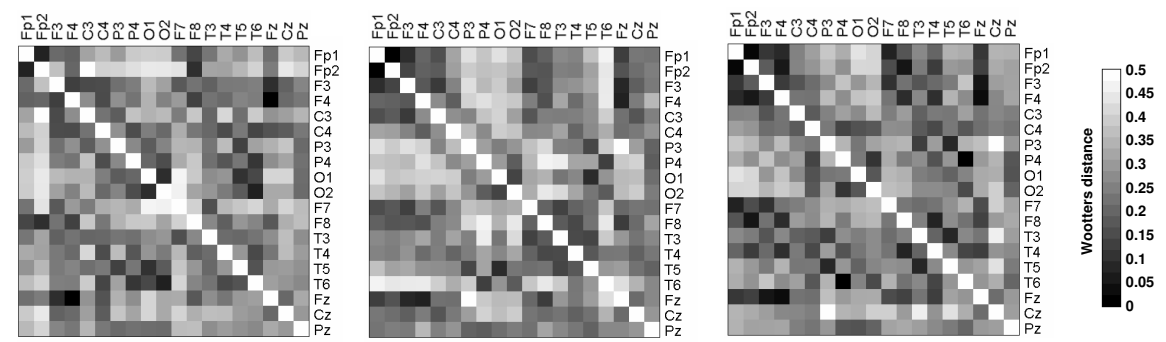

(c) Typical seizure episode signals
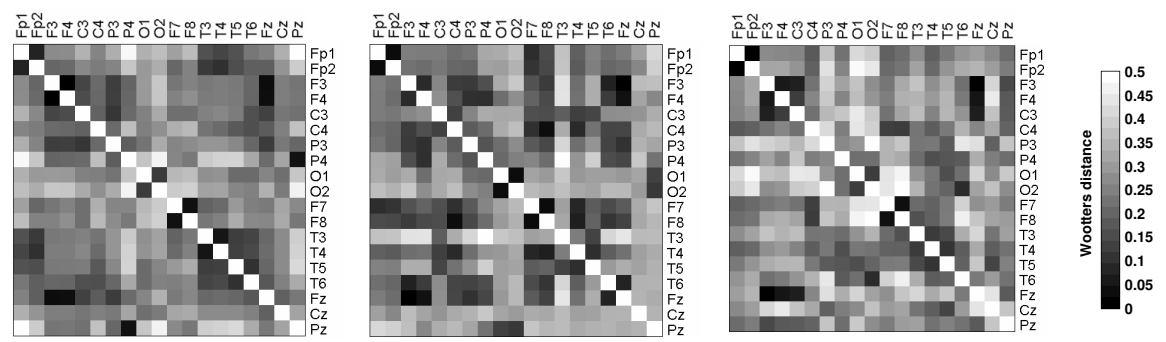

(d) Averages over all epochs Controls background

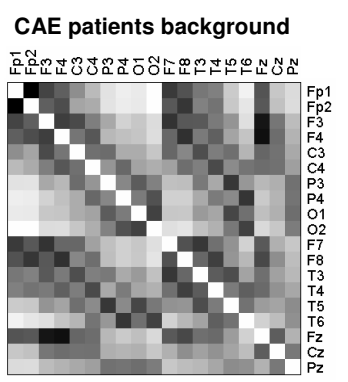

Seizure episodes
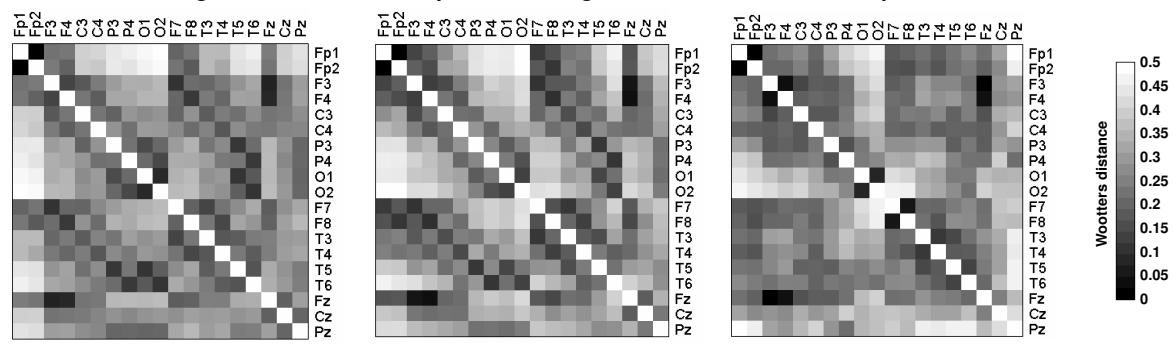

Fig. 1. Typical pixel-matrices obtained using the novel distance quantifiers between EEG channels' wavelet energy probability distribution. (a-c) Three matrices are shown for each type of signal - control background, CAE background and CAE seizure episode. The order of the EEG channels is the same in all matrices and follows the sequence in the 10-20 system. Each graphic corresponds to the average of the time-windows within a specific epoch that was chosen to be representative of the physiological state. Darker pixels indicate smaller distances (high similarity between EEG signals) and lighter pixels mean larger distances (low similarity). The matrices correspond to typical (a) healthy control individuals; (b) CAE patients; (c) seizure episodes. (d) Average matrices for the types of signals calculated using all time windows available (41 for controls background; 15 for CAE patients background; and 8 for seizure episodes). 
(a) Separation $S_{x}$ betw een classes after PCA

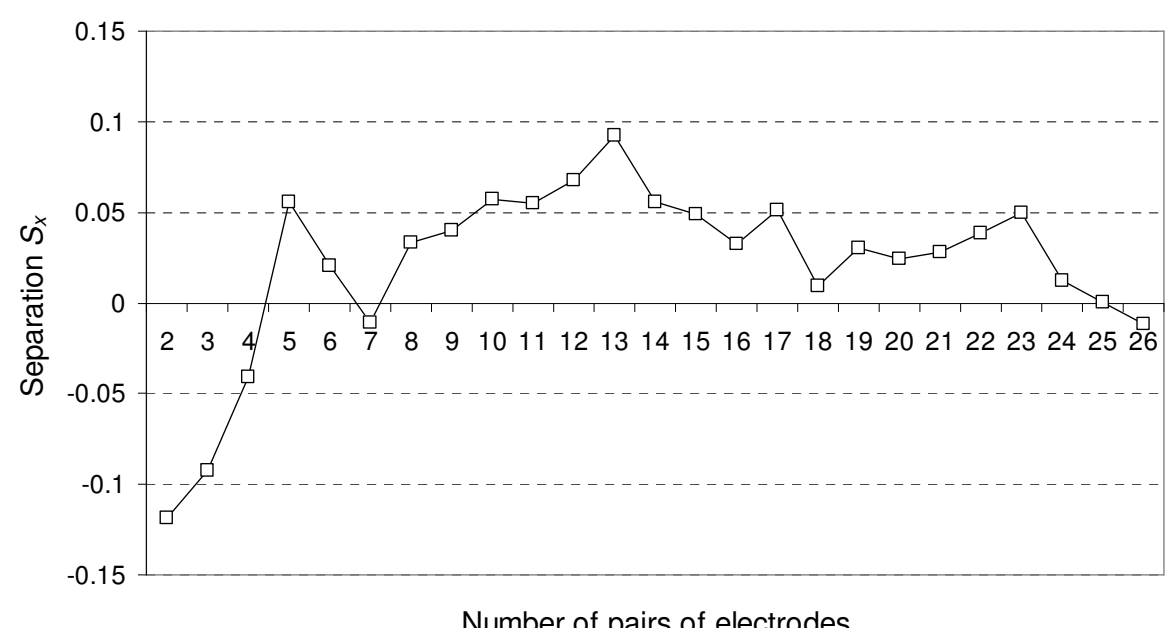

(b) Separation $S_{A H C}$ between classes after PCA

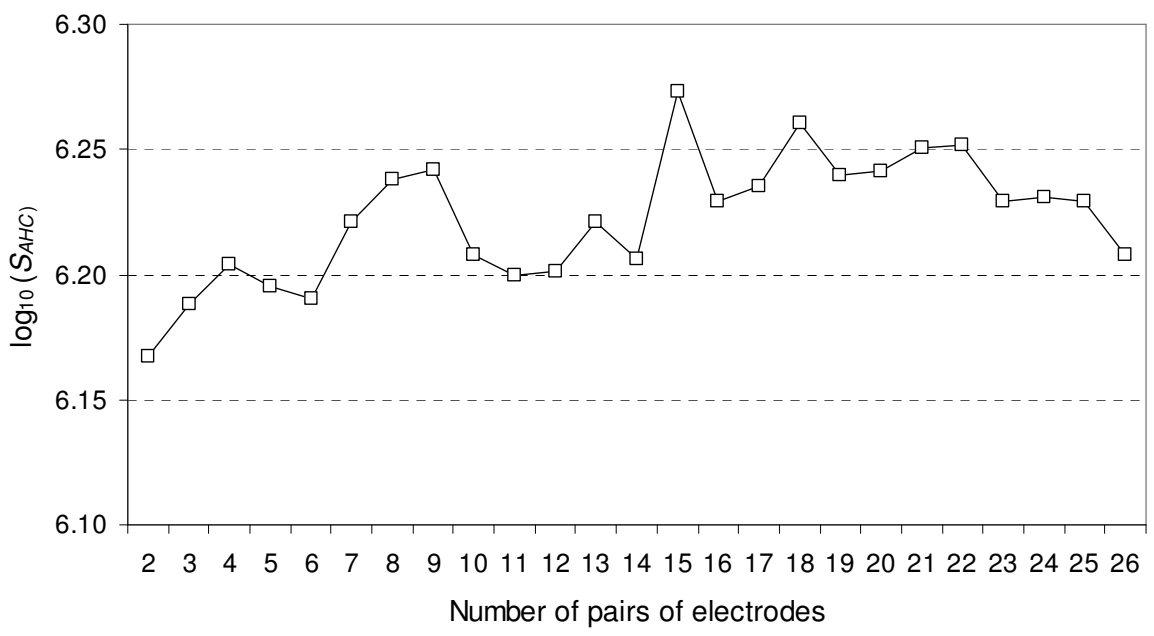

Fig. 2. Separations between healthy controls and CAE patients after the PCA, for different numbers of pairs of electrodes. Two separation measures were used - (a) $S_{x}$ and (b) $S_{A H C}$. The best separations come with the PCA calculated using the 13 pairs of electrodes with the lowest $p$-values (10 different electrodes in total) for $S_{x}$; and 15 pairs of electrodes for $S_{A H C}$ (the same 10 individual electrodes are present). Please note that the numerical values of $S_{x}$ and $S_{A H C}$ are in different scales due to the distinct calculations and thus are not directly comparable. The values of $S_{A H C}$ are in the order of $10^{6}$ and graph (b) shows the log-transformed values of $S_{A H C}$ for better visualization. However, when used in conjunction, the two measures $S_{x}$ and $S_{A H C}$ allow to identify configurations which yield consistently better separations between CAE patients and controls. Also, please note that in the $S_{x}$, negative values indicate an overlap between the classes. On the other hand, in the $S_{A H C}$ values are always positive, since it is calculated as a summation of distances between points. For more details, we refer the reader to the text. 


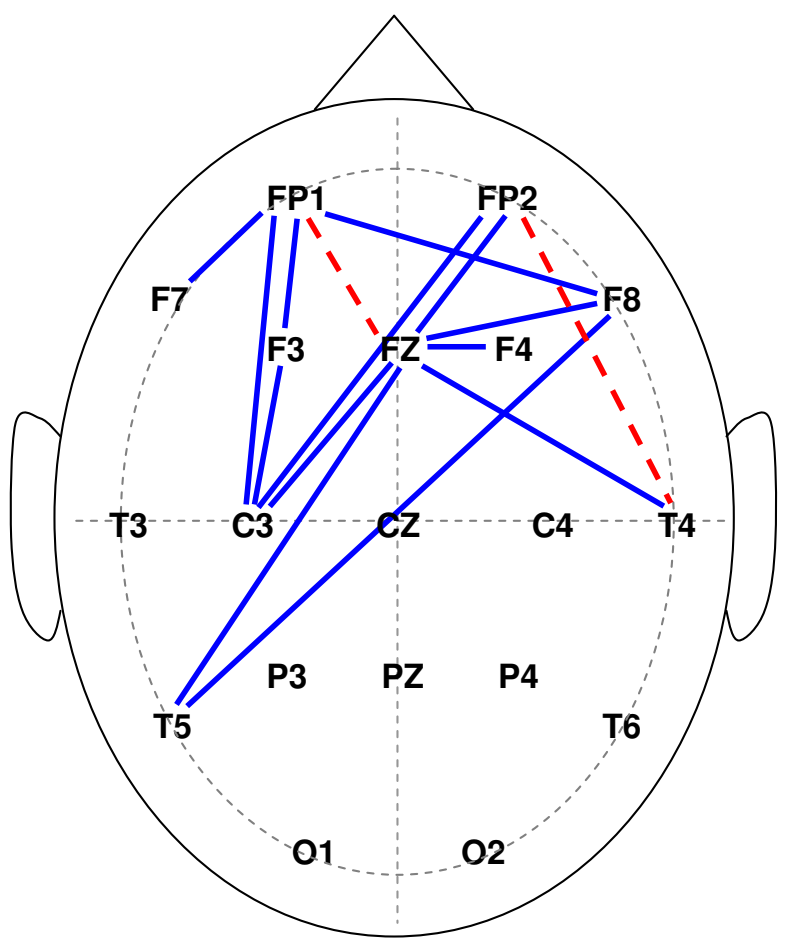

Fig. 3. Diagram showing the location of the 20 electrodes used in the CAE study. The solid lines indicate the pairs of electrodes common to the solutions with 13 pairs (best separation for $S_{x}$ measure) and 15 pairs (best separation for $S_{A H C}$ measure). The dashed lines indicate the two extra pairs of electrodes present only in the 15 pairs solution. Lines indicate a similar behaviour between the electrodes connected. They are thus an indicative of the functional networks that can differentiate the background of CAE patients and control individuals. 

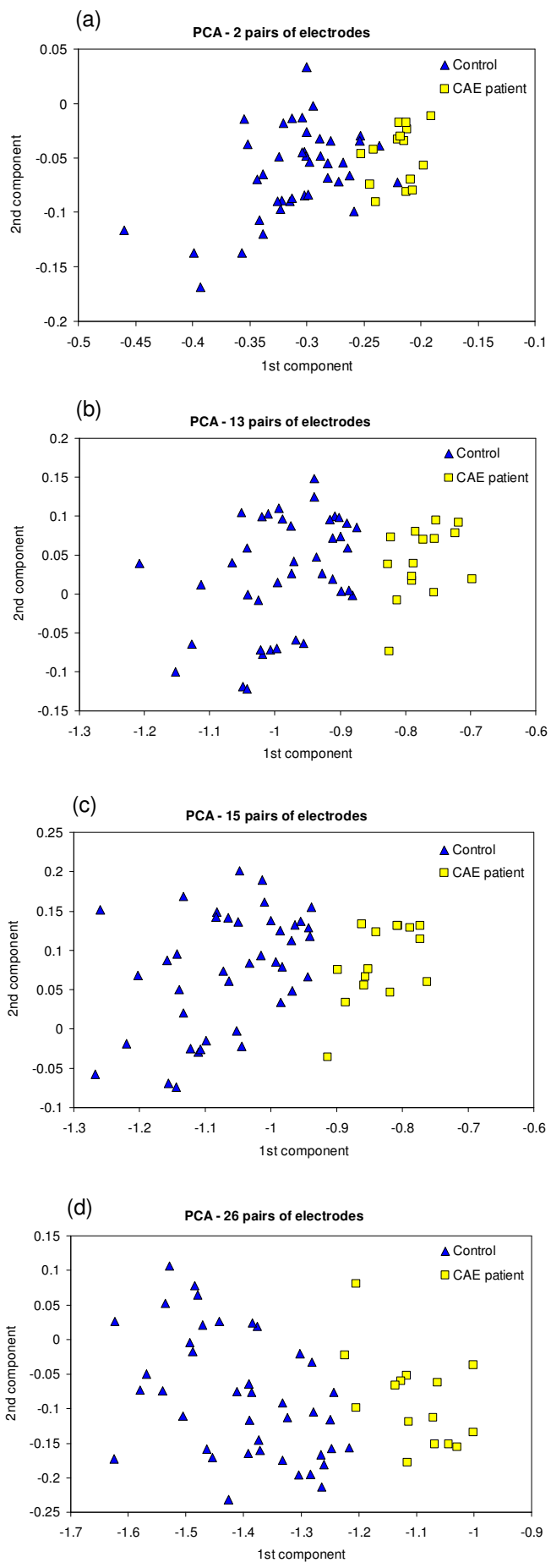

Fig. 4. PCA results for the healthy controls and CAE patients samples using different numbers of pairs of electrodes. (a) PCA of the samples using the two pairs of electrodes with best $p$-values $\left(p=2.6 \times 10^{-7}\right.$ and $\left.p=7.4 \times 10^{-7}\right)$. Notice the overlap between the two classes. (b) PCA using the 13 pairs of electrodes with best $p$-values (best separation for $S_{x}$ measure). The worst pair has $p=7.9 \times 10^{-6}$.(c) PCA using the 15 pairs of electrodes with best $p$-values (best separation for $S_{A H C}$ measure). The worst pair has $p=8.6 \times 10^{-6}$. (d) PCA using the 26 pairs of electrodes with best $p$-values (worst pair has $p=6.2 \times 10^{-5}$ ). Notice the occurrence of overlapped samples again. 